\title{
30. GEOCHEMISTRY OF COCOS PLATE PELAGIC-HEMIPELAGIC SEDIMENTS IN HOLE 487, DEEP SEA DRILLING PROJECT LEG 66 ${ }^{1}$
}

\author{
Jeremy K. Leggett, Department of Geology, Imperial College of Science \& Technology, London SW7 2BP, U.K.
}

\section{INTRODUCTION}

The sedimentary sequence recovered from Hole 487 documents northeast seafloor spreading on the Cocos Plate. A basal 65-meter unit of upper Miocene-Pliocene brown clay was deposited on the subsiding east flank of the East Pacific Rise. The overlying 105-meter unit of gray hemipelagic silt and mud was deposited when Site 487 drifted within reach of terrigenous sediment derived from the Mexican continental margin.

Analyses of 21 elements in 45 samples, taken at regular intervals up Hole 487, give a geochemical profile which shows metal enrichment in basal sediment similar to that observed in previously recovered basal sediment sections in the Eastern Pacific (e.g., von der Borch \& Rex, 1970; von der Borch et al., 1971). This verifies that the basalt cored at the base the hole is oceanic basement, since the first sediment to be deposited on newly formed ocean crust is characteristically enriched in metals, particularly $\mathrm{Fe}$ and $\mathrm{Mn}$. The metals precipitate from circulating hydrothermal-exhalative solutions which are an integral part of active ridge volcanism (summary in Jenkyns, 1978). In this chapter I discuss the geochemistry of the basal metalliferous sediment from Hole 487.

The geochemical data may also prove useful in studies of onshore volcanism: mass-balance calculations show that much (possibly all) of the incoming Cocos Plate sediment has been subducted since the Miocene (Watkins et al., this volume). It may have contributed to the genesis of magmas erupted in the Mexican volcanic arc.

\section{ANALYTICAL PROCEDURES}

Dried sediment samples were digested in hydrofluoric-perchloric acid and analyzed on an inductively coupled plasma spectrometer (A.R.L. 34000) capable of simultaneous interelement interference corrections.

\section{LITHOLOGY}

The Quaternary mud is a homogeneous grayish olive deposit with local indistinct millimeter-scale, darker parallel laminations and common ash beds. The mud itself is vitric in the 96 to 97.5 meter interval. Local mottling suggests bioturbation. The presence of reworked calcareous microfossils from 0 to 77 meters, a small

\footnotetext{
${ }^{1}$ Initial Reports of the Deep Sea Drilling Project, Volume 66.
}

shell fragment at 58 meters, and a wood fragment at 86.5 meters, together with the local lamination, suggest that much of the mud may be redeposited, probably as suspension load from large trench-overtopping turbidity flows.

The underlying brown clay is predominantly moderate to dusky yellowish brown, in places dusky red to dark reddish brown, and commonly variegated with orange areas. Zeolite micronodules and millimeter-scale better-lithified nodular developments are common. At 134 meters a $70-\mathrm{cm}$ pale olive clay interbed has a sharp contact with underlying brown clay. At 160 meters color change from grayish brown above to dusky brown below is caused by a concentration in the latter of amorphous grains of isotropic material, probably $\mathrm{Fe}$ oxide, up to several tens of microns in diameter.

Rare reworked calcareous microfossils occur at the very base of the brown clay, but carbonate determinations were below the detection limit throughout the hole (i.e., $<0.05 \% \mathrm{CaCO}_{3}$ ) and all the sediment must have accumulated below the calcite compensation depth. Hence it is not necessary to correct analyses for carbonate content.

In the basal 3 meters small angular fragments of basalt are mixed in the brown clay.

\section{RESULTS}

Fe and Mn are both significantly enriched in the basal 15 meters or so, reaching maxima of $20.4 \%$ and $9.8 \%$, respectively (Table 1 ). An anomalously high concentration $(19 \%)$ of Mn occurs at 144 meters and may reflect incorporation of a manganese micronodule in the sample. A similarly anomalous high Fe total $(27 \%)$ occurs in the top meter of gray clay; together with a very high Mo analysis (1006 ppm), this probably reflects a $1 \%$ component of heavy minerals observed in smear slides taken at this level.

The high $\mathrm{Fe}$ and $\mathrm{Mn}$ values in basal sediment are complemented by $\mathrm{Al}$ and $\mathrm{Ti}$ values which are lower than those in the younger brown clay (Fig. 1). Relative impoverishment of $\mathrm{Al}$ and $\mathrm{Ti}$ is a widely recorded characteristic of metalliferous sediments (e.g., references in Table 2).

The high $\mathrm{Fe}$ and $\mathrm{Mn}$ content of metalliferous sediments also characteristically follows with high concentrations of certain trace elements, principally metals (summary in Boström, 1973). At Site 487 brown clay, $\mathrm{Cu}, \mathrm{P}$, and $\mathrm{Sr}$ show relative enrichment in the basal 15 
Table 1. Geochemistry of Cocos Plate hemipelagic-pelagic sediments in Hole 487, DSDP Leg 66. (Analyses of $\mathrm{Al}$ to $\mathrm{P}$ in \%, Ba to V in ppm.)

\begin{tabular}{|c|c|c|c|c|c|c|c|c|c|c|c|c|c|c|c|c|c|c|c|c|c|c|c|}
\hline $\begin{array}{l}\text { Sample } \\
\text { No. }\end{array}$ & $\begin{array}{c}\text { Sample } \\
\text { (interval in } \mathrm{cm} \text { ) }\end{array}$ & Al & $\mathrm{Ca}$ & $\mathrm{Fe}$ & $\mathrm{Mn}$ & $\mathrm{Mg}$ & $\mathrm{Na}$ & K & $\mathrm{Ti}$ & $\mathbf{P}$ & $\mathrm{Ba}$ & Co & $\mathrm{Cr}$ & $\mathrm{Cu}$ & La & Li & $\mathrm{Ni}$ & Mo & $\mathrm{Pb}$ & $\mathrm{Sr}$ & $\mathrm{Zn}$ & V & $\begin{array}{l}\text { Depth } \\
\text { Sub-bottom } \\
\text { (m) }\end{array}$ \\
\hline 1 & $1-1,139-143$ & 6.32 & 0.72 & 27.32 & 0.41 & 0.99 & 1.08 & 1.34 & 0.26 & 0.06 & 536 & 26 & 216 & 83 & 14 & 52 & 69 & 1006 & 191 & 165 & 306 & 171 & 1.4 \\
\hline 2 & $2-2,32-36$ & 8.20 & 1.22 & 4.96 & 0.16 & 1.68 & 1.96 & 1.17 & 0.47 & 0.08 & 1757 & 22 & & 86 & 23 & 69 & 67 & 27 & 126 & 198 & 157 & 204 & 2.8 \\
\hline 3 & $2-5,100-104$ & 10.29 & 1.10 & 5.17 & 0.13 & 1.38 & 1.92 & 1.58 & 0.46 & 0.08 & 665 & 21 & 117 & 71 & 28 & 87 & 52 & 15 & 97 & 155 & 157 & 181 & 6.0 \\
\hline 4 & $3-3,52-56$ & 5.40 & 0.54 & 5.66 & 0.19 & 1.14 & 1.35 & 0.68 & 0.25 & 0.07 & 2163 & 19 & 105 & 81 & 28 & 47 & 62 & 21 & 128 & 146 & 155 & 125 & 14.0 \\
\hline 5 & $5-2,94-98$ & 8.89 & 1.32 & 5.12 & 1.39 & 1.66 & 1.88 & 1.18 & 0.46 & 0.10 & 831 & 23 & 89 & 114 & 22 & 74 & 84 & 31 & 101 & 186 & 188 & 229 & 31.9 \\
\hline 6 & $6-3,60-65$ & 8.36 & 0.89 & 5.08 & 0.29 & 1.57 & 1.63 & 1.15 & 0.43 & 0.08 & 962 & 23 & 68 & 106 & 24 & 74 & 83 & 23 & 94 & 164 & 238 & 212 & 42.6 \\
\hline 7 & $7-2,66-71$ & 8.83 & 0.94 & 5.25 & 0.50 & 1.58 & 1.65 & 1.32 & 0.43 & 0.07 & 994 & 23 & 74 & 119 & 24 & 75 & 92 & 25 & 88 & 167 & 199 & 211 & 50.7 \\
\hline 8 & $8-1,69-73$ & 8.40 & 1.07 & 5.46 & 0.66 & 1.73 & 2.22 & 1.26 & 0.43 & 0.09 & 1983 & 24 & & 166 & 22 & 74 & 124 & 24 & 102 & 184 & 251 & 208 & 58.7 \\
\hline 9 & $9-2,100-105$ & 9,00 & 1.01 & 5.96 & 0.47 & 1.73 & 1.50 & 1.33 & 0.47 & 0.09 & 1382 & 26 & 87 & 175 & 26 & 77 & 146 & 28 & 112 & 192 & 314 & 236 & 70.0 \\
\hline 10 & $9-5,38-43$ & 8.93 & 1.44 & 5.64 & 0.22 & 1.71 & 2.30 & 1.34 & 0.30 & 0.11 & 1379 & 23 & 123 & 151 & 23 & 82 & 125 & 27 & 92 & 195 & 207 & 218 & 73.8 \\
\hline 11 & $10-4,64-68$ & 9.12 & 0.91 & 6.03 & 0.16 & 1.73 & 1.57 & 1.30 & 0.47 & 0.08 & 1292 & 27 & 78 & 172 & 26 & 80 & 133 & 28 & 101 & 198 & 217 & 246 & 81.6 \\
\hline 12 & $11-1,98-102$ & 8.49 & 0.84 & 5.66 & 0.08 & 1.61 & 2.00 & 1.32 & 0.40 & 0.06 & 1321 & 27 & 76 & 190 & 27 & 78 & 169 & 21 & 101 & 174 & 274 & 228 & 87.5 \\
\hline 13 & $11-4,72-75$ & 8.38 & 0.89 & 6.13 & 0.06 & 1.62 & 1.70 & 1.31 & 0.38 & 0.08 & 926 & 23 & 100 & 205 & 36 & 83 & 204 & 13 & 62 & 209 & 230 & 254 & 91.7 \\
\hline 14 & $12-1,22-25$ & 8.22 & 1.01 & 6.95 & 0.10 & 1.67 & 2.11 & 1.37 & 0.39 & 0.09 & 2569 & 25 & 83 & 255 & 40 & 74 & 168 & 38 & 166 & 221 & 246 & 209 & 96.2 \\
\hline 15 & $12-1,99-104$ & 8.22 & 0.94 & 5.95 & 0.11 & 1.53 & 2.17 & 1.46 & 0.38 & 0.08 & 1757 & 34 & 77 & 518 & 39 & 72 & 266 & 19 & 85 & 196 & 276 & 258 & 97.0 \\
\hline 16 & $12-3,59-63$ & 6.67 & 1.23 & 7.94 & 0.86 & 1.98 & 2.53 & 1.08 & 0.32 & 0.08 & 2184 & 58 & 57 & 645 & 34 & 62 & 397 & 27 & 100 & 204 & 364 & 308 & 99.6 \\
\hline 17 & $13-1,7-12$ & 8.56 & 0.87 & 6.85 & 0.15 & 1.80 & 2.09 & 1.20 & 0.39 & 0.13 & 3399 & 39 & 132 & 185 & 50 & 70 & 122 & 15 & 74 & 229 & 188 & 198 & 105.6 \\
\hline 18 & $13-3,6-9$ & 8.07 & 1.31 & 7,11 & 0.10 & 1.84 & 2.39 & 1.18 & 0.39 & 0.18 & 4706 & 40 & 132 & 251 & 56 & 57 & 128 & 16 & 97 & 281 & 203 & 199 & 108.6 \\
\hline 19 & $14-2,20-25$ & 5.61 & 1.27 & 13.23 & 3.66 & 1.60 & 1.86 & 0.94 & 0.30 & 0.37 & 5604 & 92 & 94 & 585 & 107 & 35 & 371 & 25 & 129 & 289 & 262 & 501 & 116.7 \\
\hline 20 & $14-3,24-28$ & 4.99 & 1.14 & 12.04 & 7.40 & 1.57 & 1.65 & 0.72 & 0.27 & 0.34 & 4955 & 50 & & 509 & 82 & 32 & 259 & 21 & 138 & 267 & 241 & 467 & 118.2 \\
\hline 21 & $15-1,79-83$ & 4.88 & 0.72 & 11.45 & 2.28 & 1.71 & 2.69 & 0.78 & 0.23 & 0.18 & 2662 & 51 & 109 & 453 & 57 & 29 & 244 & 26 & 90 & 311 & 217 & 259 & 125.3 \\
\hline 22 & $15-2,26-30$ & 5.03 & 0.68 & 11.18 & 1.70 & 1.59 & 1.67 & 0.97 & 0.21 & 0.17 & 2357 & 42 & 75 & 407 & 60 & 29 & 201 & 16 & 76 & 285 & 226 & 226 & 126.6 \\
\hline 23 & $15-6,99-103$ & 4.89 & 1.03 & 12.92 & 2.53 & 2.00 & 2.23 & 0.73 & 0.26 & 0.24 & 3945 & 55 & 96 & 561 & 61 & 29 & 270 & 32 & 110 & 307 & 309 & 252 & 133.0 \\
\hline 24 & $16-1,35-39$ & 4.04 & 0.68 & 13.74 & 0.42 & 2.20 & 1.75 & 0.77 & 0.22 & 0.14 & 2837 & 34 & 58 & 333 & 70 & 31 & 266 & 24 & 76 & 255 & 325 & 406 & 134.3 \\
\hline 25 & $16-1,98-102$ & 4.47 & 1.21 & 12.92 & 1.90 & 1.88 & 1.98 & 0.78 & 0.22 & 0.36 & 2528 & 49 & 98 & 492 & 91 & 26 & 242 & 23 & 101 & 335 & 238 & 208 & 135 \\
\hline 26 & $16-4,117-122$ & 4.46 & 0.76 & 10.91 & 2.07 & 1.58 & 3.25 & 0.86 & 0.20 & 0.18 & 2496 & 49 & 92 & 398 & 58 & 24 & 228 & 22 & 86 & 306 & 213 & 243 & 139.7 \\
\hline 27 & $17-1,84-89$ & 3.25 & 1.45 & 8.26 & 18.99 & 1.64 & 1.05 & 0.48 & 0.15 & 0.26 & 1640 & 38 & 106 & 598 & 45 & 21 & 188 & 31 & 135 & 210 & 299 & 199 & 144.3 \\
\hline 28 & $17-1,98-102$ & 4.77 & 0.82 & 13.22 & 2.82 & 1.72 & 1.84 & 0.74 & 0.24 & 0.23 & 1732 & 73 & 83 & 547 & 106 & 28 & 235 & 21 & 142 & 358 & 224 & 303 & 144.5 \\
\hline 29 & $17-2,69-79$ & 4.74 & 0.86 & 13.59 & 3.01 & 1.55 & 2.02 & 0.70 & 0.24 & 0.31 & 4324 & 57 & 70 & 478 & 75 & 24 & 209 & 25 & 82 & 384 & 218 & 374 & 145.7 \\
\hline 30 & $18-1,99-103$ & 4.94 & 0.83 & 10.96 & 3.26 & 1.93 & 2.50 & 0.62 & 0.25 & 0.18 & 3357 & 52 & & 324 & 65 & 33 & 127 & 48 & 83 & 333 & 238 & 217 & 154.0 \\
\hline 31 & $18-1,146-150$ & 7.06 & 1.03 & 5.41 & 1.23 & 2.38 & 1.84 & 0.50 & 0.26 & 0.09 & 1876 & 18 & & 388 & 25 & 66 & 93 & 21 & 50 & 197 & 258 & 95 & 154.5 \\
\hline 32 & $18-3,99-103$ & 4.77 & 0.70 & 11.32 & 3.94 & 2.10 & 2.25 & 0.60 & 0.20 & 0.19 & 3030 & 28 & & 594 & 50 & 51 & 153 & 50 & 84 & 279 & 302 & 304 & 157.0 \\
\hline 33 & $18-4,99-103$ & 2.88 & 0,90 & 20.36 & 8.33 & 1.72 & 1.29 & 0.65 & 0.16 & 0.48 & 3579 & 64 & & 733 & 106 & 17 & 371 & 77 & 142 & 461 & 394 & 525 & 158.0 \\
\hline 34 & $18-5,89-93$ & 2.16 & 1.08 & 17.81 & 9.81 & 2.19 & 1.32 & 0.77 & 0.12 & 0.45 & 3391 & 55 & & 698 & 83 & 18 & 449 & 75 & 131 & 437 & 392 & 350 & 158.9 \\
\hline 35 & $18-6,98-102$ & 2.14 & 1.06 & 16.81 & 8.42 & 2.07 & 1.92 & 0.76 & 0.12 & 0.47 & 4027 & 47 & 57 & 625 & 81 & 17 & 348 & 65 & 88 & 418 & 356 & 347 & 160.0 \\
\hline 36 & $19-1,61-66$ & 2.28 & 1.55 & 18.44 & 7.34 & 1.79 & 1.82 & 0.66 & 0.13 & 0.57 & 3213 & 55 & 77 & 752 & 88 & 16 & 325 & 38 & 115 & 623 & 362 & 516 & 163,1 \\
\hline 37 & $19-2,29-31$ & 2.71 & 1.92 & 17.01 & 5.10 & 1.94 & 2.20 & 0.96 & 0.13 & 0.77 & 1815 & 50 & & 620 & 94 & 20 & 339 & 36 & 103 & 579 & 329 & 379 & 164.3 \\
\hline 38 & $19-3,3-5$ & 2.09 & 1.42 & 18.73 & 6.33 & 1.88 & 1.46 & 0.70 & 0.12 & 0.46 & 5215 & 56 & & 654 & 84 & 17 & 302 & 53 & 127 & 656 & 373 & 479 & 165.5 \\
\hline 39 & $19-4,93-95$ & 2.89 & 1.27 & 15.68 & 6.26 & 2.37 & 1.59 & 1.02 & 0.15 & 0.37 & 2147 & 53 & 59 & 499 & 73 & 24 & 304 & 66 & 115 & 399 & 370 & 221 & 167.9 \\
\hline 40 & $19-5,49-53$ & 3.03 & 2.35 & 14.51 & 5.52 & 2.13 & 2.64 & 0.79 & 0.16 & 0.37 & 2776 & 45 & & 528 & 70 & 20 & 271 & 73 & 100 & 415 & 307 & 295 & 169.0 \\
\hline 41 & $19-5,78-80$ & 4.90 & 5.20 & 14.64 & 4.49 & 2.80 & 1.33 & 0.44 & 0.29 & 0.30 & 732 & 55 & 173 & 439 & 55 & 15 & 241 & 83 & 95 & 337 & 246 & 382 & 169.2 \\
\hline 42 & $19-5,149-150$ & 5.82 & 6.07 & 11.24 & 3.13 & 3.17 & 1.72 & 0.32 & 0.34 & 0.21 & 1709 & 53 & 151 & 328 & 39 & 14 & 214 & 57 & 103 & 256 & 188 & 335 & 170.0 \\
\hline 43 & $20-1,103-105$ & 4.87 & 3.58 & 14.04 & 4.07 & 2.45 & 1.64 & 0.66 & 0.27 & 0.32 & 3513 & 58 & 76 & 465 & 68 & 19 & 259 & 60 & 159 & 355 & 261 & 365 & 173.0 \\
\hline 44 & $20-2,4-6$ & 5.46 & 4.18 & 12.90 & 3.52 & 2.66 & 1.73 & 0.51 & 0.31 & 0.27 & 2811 & 60 & 159 & 423 & 57 & 17 & 231 & 55 & 84 & 292 & 227 & 348 & 173.5 \\
\hline 45 & $20-2,16-18$ & 6.89 & 6.81 & 11.04 & 2.33 & 3.25 & 1.80 & 0.35 & 0.40 & 0.18 & 3687 & 55 & 166 & 277 & 35 & 13 & 189 & 70 & 115 & 218 & 178 & 355 & 173.7 \\
\hline
\end{tabular}

meters (Table 1, Fig. 1). $\mathrm{Ba}, \mathrm{Co}, \mathrm{Cr}, \mathrm{Ni}, \mathrm{Pb}, \mathrm{Zu}$, and $\mathrm{V}$ show no significant variation down the brown clay sequence.

In the basal 5 meters the metalliferous brown clay has lower $\mathrm{Fe}$ and $\mathrm{Mn}$ than the 10 meters or so of metalliferous sediment above it; $\mathrm{Al}$ and $\mathrm{Ti}$ values are concomitantly higher (Fig. 1). This probably reflects contamination of the samples by fragments of basalt previously mentioned.

The geochemical differences between the brown clay and overlying greenish gray hemipelagic mud are reflected principally by uniformly higher values for $\mathrm{Fe}$, $\mathrm{Mn}, \mathrm{P}, \mathrm{Cu}, \mathrm{Sr}, \mathrm{Co}$, and $\mathrm{La}$ in the former and for $\mathrm{Al}, \mathrm{Ti}$, and $\mathrm{Li}$ in the latter. The yellowish gray clay interval at about 110 meters does not have a significantly different geochemistry from the hemipelagic mud.

Within the brown clay sequence high metal concentrations are probably located in the amorphous isotropic oxide grains seen under the microscope. A high concentration of these gives rise to the dusky brown color noted in the basal 12 meters. Other color change features of interest in the brown clay sequence are the $70-\mathrm{cm}$ interbedded olive clay at about 134 meters and a decimeter-scale interbed of orange-pink clay with millimeter-scale indurated areas at 154.5 meters. The former shows a markedly lower Mn content than is normal in the brown clay, though without change in the Fe level
(Sample 24, Table 1). The latter shows lower Fe and Mn and higher Al than normal brown clay (Sample 31, Table 1).

\section{ACKNOWLEDGMENT}

I would like to thank Dr. Peter Bush for reviewing the manuscript.

\section{REFERENCES}

Boström, K., 1973. The origin of ferromanganoan active ridge sediments. Stockholm Contrib. Geol., 26:149-243.

Boström, K., and Peterson, M. N. A., 1969. The origin of aluminiumpoor ferromanganoan sediments in areas of high heat flow on the East Pacific Rise. Econ Geol., 61:1258-1265.

Dymond, J., Corliss, J. B., Heath, C. W., et al., 1973. Origin of metalliferous sediments from the Pacific Ocean. Geol. Soc. Am. Bull., 84:3355-3372.

Jenkyns, H. C., 1978. Pelagic environments. In Reading, H. G. (Ed.), Sedimentary Environments and Facies: Oxford (Blackwell Scientific Publications), pp. 314-371.

Turekian, K. K., and Wedepohl, K. H., 1961. Distribution of the elements in some major units of the Earth's crust. Geol. Soc. Am. Bull., 72:175-192.

von der Borch, C. C., Nesteroff, W. D., and Galehouse, J., 1971. Iron-rich sediments cored during Leg VIII of the Deep Sea Drilling Project. In Tracey, J. I., Jr., Sutton, G. H., et al., Init. Repts. DSDP, 8: Washington (U.S. Govt. Printing Office), 829-836.

von der Borch, C., and Rex, R., 1970. Amorphous iron oxide precipitates in sediments cored during Leg V, DSDP. In McManus, D. A., Burns, R. E., et al., Init. Repts. DSDP, 5: Washington (U.S. Govt. Printing Office), 541-544. 


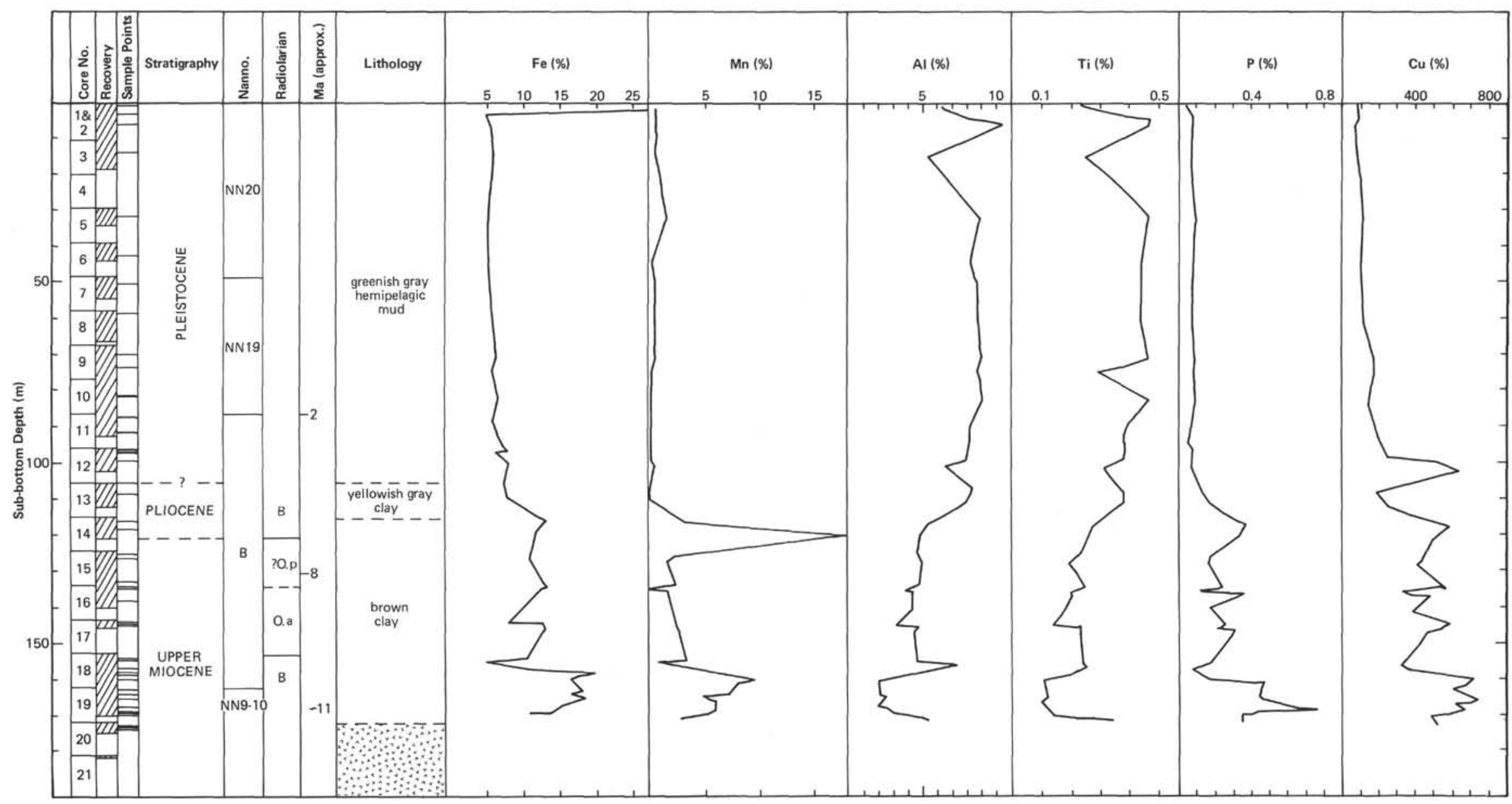

Figure 1. Geochemical profiles for $\mathrm{Fe}, \mathrm{Mn}, \mathrm{Al}, \mathrm{Ti}, \mathrm{Cu}$, and $\mathrm{P}$ in Hole 487, DSDP Leg 66, Cocos Plate. 


\section{J. K. LEGGETT}

Table 2. Geochemistry of basal Cocos Plate sediments, Hole 487: Comparison with other basal Pacific sediments and normal deep sea clay.

\begin{tabular}{|c|c|c|c|c|c|c|c|c|c|}
\hline $\begin{array}{l}\text { Cores } 18,19 \\
\text { (13 samples) }\end{array}$ & $\begin{array}{c}\mathrm{Fe} \\
14.8 \\
(5.4-20.4)\end{array}$ & $\begin{array}{c}\mathrm{Mn} \\
5.6 \\
(1.2-9.8)\end{array}$ & $\begin{array}{c}\mathrm{Al} \\
3.7 \\
(2.1-7.0)\end{array}$ & $\begin{array}{c}\mathrm{Ti} \\
0.19 \\
(0.09-0.77)\end{array}$ & $\begin{array}{c}\mathrm{Cu} \\
552 \\
(324-752)\end{array}$ & $\begin{array}{c}\mathrm{Zn} \\
317 \\
(188-394)\end{array}$ & $\begin{array}{c}\mathrm{Ba} \\
2836 \\
(732-4027)\end{array}$ & $\begin{array}{c}\mathrm{Pb} \\
103 \\
(50-142)\end{array}$ & $\begin{array}{c}\mathrm{Sr} \\
415 \\
(197-656)\end{array}$ \\
\hline $\begin{array}{l}\text { Metalliferous sediments } \\
\text { from the East Pacific Rise }{ }^{a}\end{array}$ & $\begin{array}{c}18.0 \\
(5.7-22.6)\end{array}$ & $\begin{array}{c}3.9 \\
(0.6-8.8)\end{array}$ & $\begin{array}{l}3.5 \\
(0.6-8.5)\end{array}$ & $\begin{array}{c}0.2 \\
(0.01-0.4)\end{array}$ & $\begin{array}{c}870 \\
(400-1800)\end{array}$ & $\begin{array}{c}315 \\
(190-530)\end{array}$ & n.a. & n.a. & n.a. \\
\hline $\begin{array}{l}\text { East Pacific Rise } \\
\text { and basal Pacific metalliferous sediments } b\end{array}$ & $\begin{array}{c}21.2 \\
(12.7-28.5)\end{array}$ & $\begin{array}{c}5.9 \\
(4.53-7.66)\end{array}$ & $\begin{array}{c}1.9 \\
(0.58-3.41)\end{array}$ & n.a. & $\begin{array}{c}958 \\
(600-1490)\end{array}$ & n.a. & $\begin{array}{c}5128 \\
(1500-15000)\end{array}$ & n.a. & n.a. \\
\hline Average deep sea clay ${ }^{c}$ & 6.5 & 0.7 & 8.4 & 0.46 & 250 & 160 & 100 & 68 & 180 \\
\hline
\end{tabular}

Note: Range of averaged values in parentheses. $\mathrm{Fe}$ to $\mathrm{Ti}$ as $\%, \mathrm{Cu}$ to $\mathrm{Sr}$ as $\mathrm{ppm}, \mathrm{n} . \mathrm{a}=$ = not analyzed.

a From Boström \& Peterson, 1969 (21 samples, recalculated on a carbonate-free basis).

b From Dymond et al., 1973 (11 samples).

c From Turekian \& Wedepohl, 1961. 